\title{
Abnormal mucus in cap polyposis
}

\author{
M P Buisine, J F Colombel, M Lecomte-Houcke, P Gower, J P Aubert, N Porchet, A Janin
}

Laboratoire de Biochimie et de Biologie Moléculaire, Hôpital Huriez, CHRU Lille and INSERM U377, Lille, France M P Buisine

J P Aubert

N Porchet

Clinique des Maladies de 1'Appareil Digestif et de la Nutrition, Hôpital Huriez, CHRU Lille, France

J F Colombel

P Gower

Laboratoire

d'Anatomie et

Cytologie

Pathologiques, Faculté

de Médecine, CHRU

Lille, France

M Lecomte-Houcke

Laboratoire

d'Anatomie et

Cytologie

Pathologiques, Hôpital

Calmette, CHRU Lille,

France

A Janin

Correspondence to:

N Porchet, U377 INSERM,

Place de Verdun, 59045 Lille

Cedex, France.

Accepted for publication 16 July 1997

\begin{abstract}
Background-Cap polyposis is a rare disease characterised by mucoid and bloody diarrhoea, with polyps covered by a cap of mucoid and fibrinopurulent exudate. The pathogenesis is not known.

Aims-To pour some light on cap polyposis pathogenesis, by examining the mucus of patients and analysing the expression of five mucin genes, MUC2, MUC3, MUC4, $M U C 5 A C$, and MUC5B.

Patient and methods-The study was performed on biopsy specimens taken from a patient with recurrent cap polyposis. Histochemical examination, electron microscopy, and mRNA in situ hybridisation were used.

Results-The mucus of cap polyposis differed in three respects from that of normal adult colon: abnormal ultrastructure of the mucus in the goblet cells, predominance of non-sulphated mucins, abnormal expression of the MUC4, MUC3, and MUC5AC genes.

Conclusions-Most of these abnormalities have been reported for other pathological situations, suggesting that the abnormalities observed in the mucus of this patient with cap polyposis are probably secondary phenomena rather than primary. However, the mucin abnormalities detected, which reflect deregulation of the expression of three apomucin genes, abnormal glycosylation, and abnormalities of the secretion process, are also probably involved in the clinical manifestations of cap polyposis.

(Gut 1998;42:135-138)
\end{abstract}

Keywords: cap polyposis; mucins; histochemistry; ultrastructure; in situ hybridisation

Cap polyposis is a rare disease described by Williams et al. ${ }^{1}$ It is characterised by mucoid and bloody diarrhoea, with polyps covered by a cap of mucoid and fibrinopurulent exudate. Very few cases have been reported. ${ }^{1-4}$ It has been postulated that abnormal colonic motility leading to mucosal prolapse may be an important cause. ${ }^{12}$ No specific viral or bacterial agent has been identified.

The mucus present in the dilated cystic glands and in the mucopurulent cap has not previously been analysed. We made a systematic study of the mucus of the characteristic polyps by histochemical and electron microscopic examination and looked at the expression of five mucin genes, MUC2, MUC3, $M U C 4, M U C 5 A C$, and $M U C 5 B$ (for a review, see $^{5}$ ), using in situ hybridisation, in a patient with recurrence of cap polyposis. The results

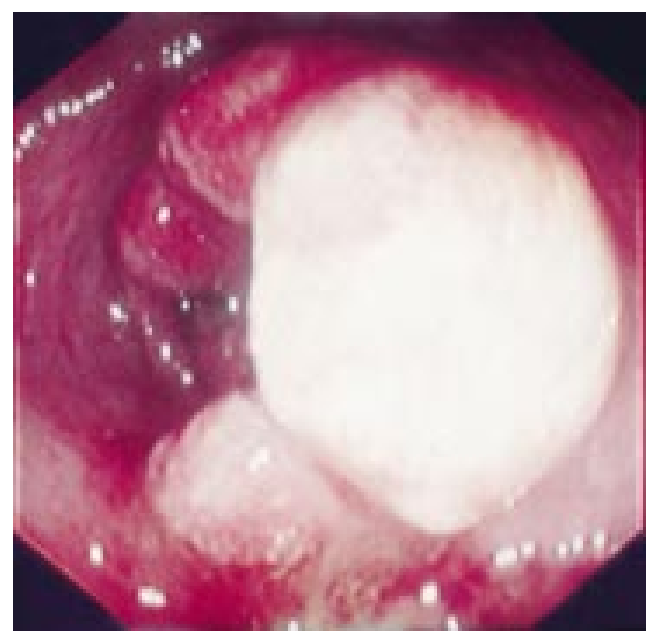

Figure 1 Recurrence of cap polyposis: endoscopic appearance of the polyps.

were compared with those found on samples of normal adult colon.

\section{Patient and methods}

PATIENT

A 42 year old woman complained of mucus and bloody diarrhoea with abdominal and rectal pain three days after operation for a uterine myoma in December 1991. A first sigmoidoscopy in January 1992 showed a flushed oedematous mucosa with abraded areas in the sigmoid colon but not in the rectum. Biopsy samples suggested non-specific inflammatory colitis. Small sessile inflammatory polyps were found $15-35 \mathrm{~cm}$ from the anal margin in following sigmoidoscopies. The patient's symptoms worsened despite treatment with 5-aminosalicylate. A sigmoid colectomy was performed in March 1993, and the diagnosis of cap polyposis was established on the basis of the appearance of the polyps, which were formed from hypertrophic colonic mucosa with dilated glands containing mucus and granulocytes, covered by a cap formed from mucus, fibrin, and leucocytes. ${ }^{3}$ The patient remained well for ten months. Recurrence of mucoid diarrhoea began in January 1994 under treatment with 5-aminosalicylate. A colonoscopy performed in March 1994 showed three small polyps located on the colorectal anastomosis (fig 1).

\section{METHODS}

The three polyps were resected and immediately cut into two halves. One half was fixed in $4 \%$ paraformaldehyde in cacodylate buffer and further processed for paraffin embedding for histological and histochemical examination and in situ hybridisation; the other one was 

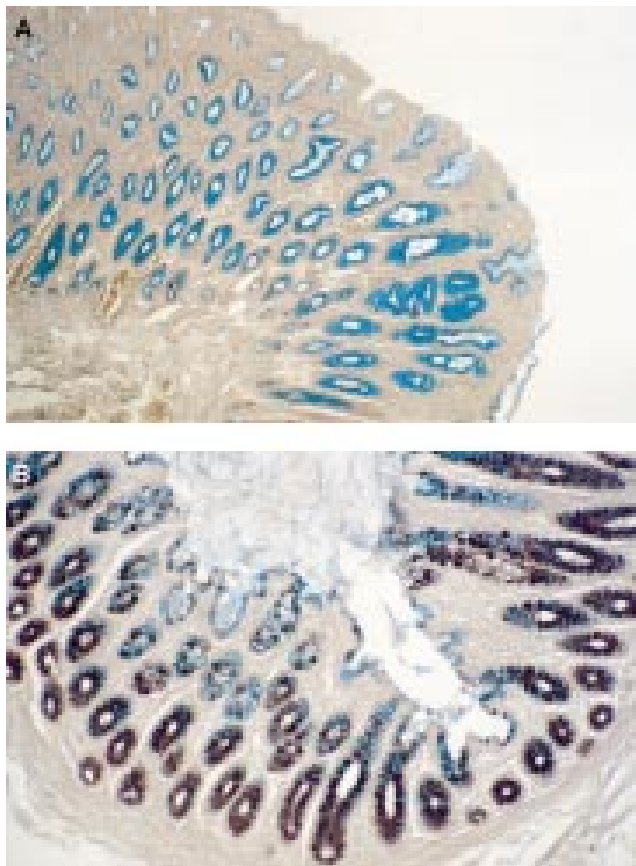

Figure 2 Histochemical examination using high iron diamine/alcian blue at $\mathrm{pH} 2.5$ of $(A)$ cap polyposis with exclusive expression of non-sulphated mucins and $(B)$ normal colon with predominance of sulphomucins.

fixed in $2 \%$ glutaraldehyde in cacodylate buffer and embedded in Epon for ultrastructural examination.

Mucin was examined histochemically as described by Spicer ${ }^{6}$ with high iron diamine/ alcian blue $\mathrm{pH} 2.5$. This method allows simultaneous analysis on the same sections of sulphomucins, which stain black, and nonsulphated mucins, which stain blue.

In situ hybridisation was performed on sequential paraffin sections using 48-mer oligonucleotides corresponding to five human mucin genes, MUC2, MUC3, MUC4, $M U C 5 A C$, and $M U C 5 B$. Because of the small size of the biopsy sample, the expression of these five genes was analysed in preference to MUC1, MUC6, and MUC7, since (a) MUC2, MUC3, MUC4, and MUC5B have been reported to be the major mucin genes expressed in the large intestine $e^{5}$ and $(b)$ we found that $M U C 5 A C$ was deregulated in rectosigmoid villous adenomas. ${ }^{7}$ These probes were labelled by addition of a tail of $\left[\alpha-\left[{ }^{35} \mathrm{~S}\right]\right.$ thio $]-$ $\mathrm{dATP}$, and used for the hybridisation procedure as previously described. ${ }^{8}$ The intensity of the hybridisation signal was scored semiquantitatively by two independent observers as: -, absent; + , weak; ++ , moderate; +++ , strong.

The ultrastructural study focused on epithelial cells and the fine structure of the mucus.

Eight samples of colonic mucosa were taken on surgical specimens at $5-10 \mathrm{~cm}$ from the carcinoma and examined in parallel. Samples were normal at histological examination.

\section{Results}

The mucus in cap polyposis differed from that of normal colon in its ultrastructural character- istics and in its composition, as ascertained by histochemical examination and in situ hybridisation.

Histochemically, the differences were found in the polyps and about $1 \mathrm{~cm}$ of the surrounding mucosa. In these areas, all the mucuscontaining epithelial cells, whether in the glands or superficial epithelium or the mucoid superficial cap, stained only for non-sulphated mucins (fig 2A). The same areas were negative for sulphomucins. In normal colonic mucosa,
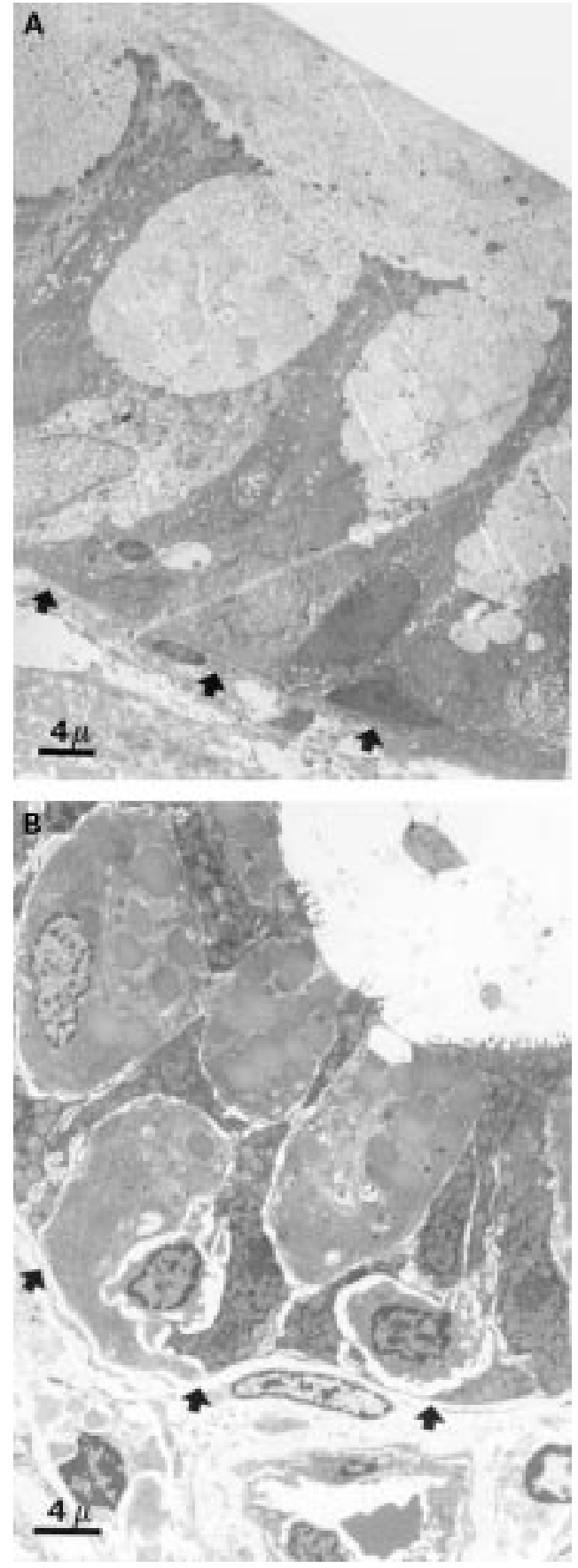

Figure 3 Transmission electron microscopy of colonic epithelium. (A) Cap polyposis: the thick superficial layer of mucus is in continuity with the mucus of goblet cells, but does not adhere to the cytoplasmic membrane of epithelial cells; mucus droplets coalesce. (B) Normal colon: the mucus is only found within the goblet cells; well formed mucus droplets are found from the bottom to the apex of the goblets. 
Table 1 Expression of the mucin genes in cap polyposis and normal colon

\begin{tabular}{llllll}
\hline \multirow{2}{*}{ Tissues } & \multicolumn{2}{l}{ Probes } & & & \\
\cline { 2 - 6 } & MUC2 & MUC3 & MUC4 & MUC5AC & MUC5B \\
\hline Cap polyposis & $\mathrm{S}:+++\star$ & $\mathrm{S}:+$ & $\mathrm{S}:+++$ & $\mathrm{S}:++$ & $\mathrm{S}:-$ \\
Normal colon $(\mathrm{n}=8)$ & $\mathrm{C}:+++$ & $\mathrm{C}:-$ & $\mathrm{C}:+++$ & $\mathrm{C}:-$ & $\mathrm{C}:-$ \\
& $\mathrm{S}:+++$ & $\mathrm{S}:+++++$ & $\mathrm{S}:++++$ & $\mathrm{S}:-$ & $\mathrm{S}:-$ \\
& $\mathrm{C}:+++$ & $\mathrm{C}:-$ & $\mathrm{C}:+++$ & $\mathrm{C}:-$ & $\mathrm{C}:-$ \\
\hline
\end{tabular}

S, surface epithelium; C, crypt epithelium.

${ }^{\star}$ Labelling intensity: - , absent; + , weak; ++ , moderate; +++ , strong.

†Focal labelling. polyps. Table 1 summarises the results. The MUC2 probe gave the strongest labelling. MUC2 mRNAs were observed in the perinuclear region of goblet cells. A strong signal was observed with the MUC4 probe in all epithelial cells on the polyps (fig 4A). MUC5AC gave a weak signal in some of the surface epithelial cells. A competition study with a 50-fold excess of the same unlabelled oligonucleotide checked the quality of this signal.

MUC3 gave a very weak signal in all cells throughout the epithelium - that is, goblet cells and absorptive cells. MUC5B was not detected.

On normal colonic controls, data were in accordance with those obtained in previous studies. ${ }^{78}$ The $M U C 2$ probe gave the strongest labelling. It was observed in the perinuclear region of the goblet cells, but with the MUC4 probe, all the epithelial cells were only moderately labelled (fig 4B). MUC3 showed homogeneous strong labelling of all epithelial cells, which was located at the surface and in the upper part of the crypts of Lieberkuhn. $M U C 5 B$ and $M U C 5 A C$ were not detected in normal colonic mucosa by in situ hybridisation.

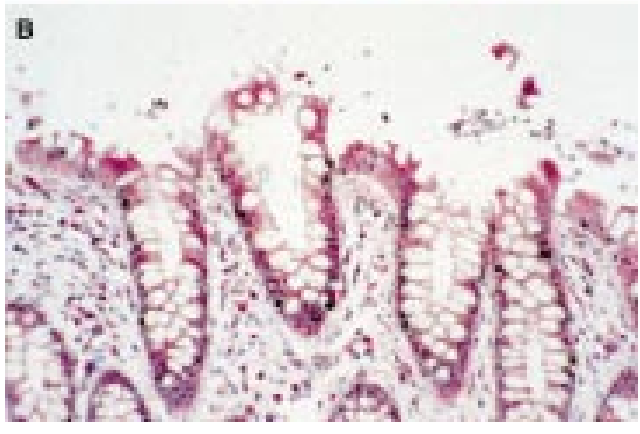

Figure 4 In situ hybridisation with the MUC4 probe using methyl green pyronin counterstain. (A) Cap polyposis: strong labelling of all epithelial cells; (B) normal colon: moderate labelling of all epithelial cells.

both non-sulphated and sulphomucins were expressed in the goblet cells, with a predominance of sulphomucins (fig 2B). Sulphomucins were more abundant in the lower part of the glands and non-sulphated mucins were present in the upper part.

Under the electron microscope, the epithelial cells of the polyp mucosa were covered by a thick layer of mucus on their surface. This mucus layer was in close continuity with the mucus located at the apical pole of the goblet cells. However, this superficial mucus layer showed no sign of adhesion to the cytoplasmic membrane of the epithelial cells (fig 3A).

All mucus goblets were hypertrophied. They contained well formed mucus droplets only in their lower part. In the upper part of the mucus goblets, mucus droplets coalesced and were extruded in conglomerated masses. In the thick layer covering the epithelium, the mucus was plainly and uniformly distributed.

In the normal adult colon, no thick and continuous mucus layer was visualised covering the epithelium. The mucus goblets were smaller and of more irregular size and shape, and mucus droplets were found from the bottom to the apex of the goblets (fig 3B).

In situ hybridisation was used to study the expression of five different mucin genes in the

\section{Discussion}

In the present study of the mucus in a case of cap polyposis, we found differences in the histochemistry, ultrastructure, and mucin gene expression when compared with normal colonic mucosa.

Histochemical analysis of the mucus in cap polyposis showed exclusive expression of nonsulphated mucins in the characteristic polyps and in the adjacent mucosa, while both non-sulphated and sulphated mucins were expressed in normal colonic mucosa. A similar predominance of non-sulphated mucins has been reported as characteristic of "transitional mucosa" associated with colorectal adenocarcinoma by Filipe..$^{9-11}$ However, further studies also found a predominance of non-sulphated mucins in other types of colorectal carcinomas, ${ }^{12}$ as well as in familial polyposis, ${ }^{13}$ colorectal adenomas, ${ }^{11}$ and ulcerative colitis with dysplastic changes. ${ }^{14} 15$ It would thus appear that a predominance of non-sulphated mucins is found in areas of colonic mucosa associated with a wide range of pathological situations.

Ultrastructurally, the characteristic cap found on the polyps in cap polyposis was composed of a thick layer of mucus. The adhesion of this mucus layer to the underlying epithelium was mediated by a close continuity with the mucus of goblet cells, but not by adhesion to the cytoplasmic membrane of epithelial cells. The adhesive properties of this mucus might be linked to abnormalities in the packaging of mucus droplets, since, in this case of cap polyposis mucus, droplets coalesced and were extruded in conglomerated masses. This phenomenon may also be related to the abnormal biochemical composition of this mucus and/or altered structure of the mucins. This was not found in biopsy specimens of normal colonic mucosa. However, similar packaging abnormalities have 
been reported in the ultrastructural study of six cases of "transitional mucosa" adjacent to colonic carcinomas. ${ }^{16}$

The expression of the five mucin genes studied by in situ hybridisation also differed in this case of cap polyposis from normal colonic mucosa. MUC4 was expressed at a high level in the polyps in cap polyposis, whereas it is expressed only at a moderate level in normal mucosa. ${ }^{78}$ So far, the only known pathological condition to show such an overexpression of MUC4 is ductal pancreatic adenocarcinomas. ${ }^{17}$

After $M U C 2, M U C 3$ is the predominant mucin gene expressed in normal colon as well as in normal small intestine. ${ }^{78}$ In this case of cap polyposis, MUC3 was clearly poorly expressed compared with the situation in normal colon. The MUC3 gene has been observed to be deregulated in other pathological situations. In colon cancers, a discrepancy is observed, since MUC2 and MUC3 are expressed at a high level in colloid cancers ${ }^{18}$ and at a low level in other types of colon cancer. ${ }^{18}{ }^{19}$ MUC3 has been reported to be expressed at a very low level in ulcerative colitis, ${ }^{20}$ as in this case of cap polyposis. Interestingly, a case has been recently reported of a patient with cap polyposis who had been treated for ulcerative colitis for seven years. ${ }^{4}$ The data, taken together, suggest that a common mechanism might occur in the pathogenesis of cap polyposis and ulcerative colitis.

MUC5AC is abnormally expressed in a few cells in this case of cap polyposis. It is normally expressed in both bronchial and gastric superficial epithelium, but not in normal intestinal mucosae..$^{78}$ We recently reported aberrant expression of $M U C 5 A C$ in rectosigmoid villous adenomas. $^{7}$ Moreover, we showed that MUC5AC mRNA expression decreased as the grade of dysplasia advanced, suggesting that $M U C 5 A C$ gene expression is an early event in the multistep process of colonic tumorigenesis. ${ }^{7}$ The finding of $M U C 5 A C$ in fetal intestine at an early stage of development (unpublished observation) reinforced our suggestion that the expression of $M U C 5 A C$ is linked to the degree of intestinal epithelial cell differentiation. The expression of $M U C 5 A C$ in a few cells in this case of cap polyposis might reflect an abnormal differentiation process.

Thus cap polyposis secretes mucus in abundance, but this mucus is qualitatively abnormal. The ultrastructural features of the mucus in the goblet cells is also altered. Mucus in cap polyposis differs from that of normal colon by quantitative disequilibrium between the expressed apomucins, by a large predominance of non-sulphated mucins and a large decrease in sulphated mucins. Unfortunately, so far nothing is known about the relationship between glycosylation, as studied by histochemistry, and the different apomucins. The most likely explanation for the lack of sulphomucins in cap polyposis is a reduction in sulphotransferase activity, leading to dramatic changes in the carbohydrate chains and charge of mucin gene products. The abundant secreted mucus may be a direct consequence of the overexpression of MUC4 and MUC5AC.
In conclusion, we have shown that the mucus in this case of cap polyposis differs from that of normal colon. Our data failed to show whether there is direct involvement of mucins in the initial steps of the pathogenesis of cap polyposis. Non-typical and specific changes in mucins, as observed by histochemical and electron microscopic examination and in situ hybridisation, were detected. Thus we suggest that these alterations are probably a secondary rather than a primary phenomenon. Nevertheless, it is noteworthy that most of these changes have been described in preneoplastic colonic mucosa-for example, transitional mucosa, adenomas. Whatever the mechanism involved, abnormalities detected in the mucus composition and ultrastructure in cap polyposis are likely to have important consequences for mucosal protection and to be involved in the clinical manifestations of cap polyposis. This work was supported by grants from the Association pour la
Recherche sur le Cancer, INSERM (CRI number 4U004B), Recherche sur le Cancer, INSERM (CRI number 4U004B),
and the CH\&U de Lille (PHRC 1994, contracts 96/09.29/9595 and the CH\&U de Lille (PHRC 1994, contracts 96/09.29/9595 and $96 / 38 / 9713)$. The authors would like
Dieu for excellent technical assistance.

1 Williams GT, Bussey HR, Morson BC. Inflammatory "cap" polyps of the large intestine. Br $\mathcal{f}$ Surg 1985;72(suppl):S133.

2 Campbell AP, Cobb CA, Chapman RWG, et al. Cap polyposis: an unusual cause of diarrhoea. Gut 1993;34: 562-4.

3 Genehot M, Colombel JF, Wolschies E, et al. Cap polyposis occurring in the postoperative course of pelvic surgery. Gut

4 Oshitani N, Morlyama Y, Matsumoto T, et al. Protein-losing enteropathy from cap polyposis. Lancet 1995;346:1567.

5 Lesuffleur T, Zweibaum A, Real FX. Mucins in normal and neoplastic human gastrointestinal tissues. Crit Rev Oncol Hematol 1994;17:153-80.

6 Spicer SS. Diamine methods for differentiating mucosubstances histochemically. $\mathcal{F}$ Histochem Cytochem 1965;13: 211-34

7 Buisine MP, Janin A, Maunoury V, et al. Aberrant expression of a human mucin gene $(M U C 5 A C)$ in rectosigmoid villous adenoma. Gastroenterology 1996;110:84-91.

8 Audie JP, Janin A, Porchet N, et al. Expression of human mucin genes in respiratory, digestive, and reproductive tracts ascertained by in situ hybridisation. $\mathcal{F}$ Histochem Cytochem 1993;4:1479-85.

9 Filipe MI. Value of histochemical reactions for mucosubstances in the diagnosis of certain pathological conditions of the colon and rectum. Gut 1969;10:577-86.

10 Filipe MI. Mucins in the human gastrointestinal epithelium: a review. Investigative Cell Pathology 1979;2: 195-216.

11 Filipe MI. Mucins of normal, premalignant and malignant colonic mucosa. In: Wolman SR, Mastromarino AJ, ed. Progress in cancer research and therapy. New York: Raven Press, 1984:237-51

12 Isaacson P, Attwood PRA. Failure to demonstrate specificity of the morphologic and histochemical changes in mucosa adjacent to colonic carcinoma (transitional mucosa). $\mathcal{F}$ Clin Pathol 1979;32:214-18.

13 Filipe MI, Mughal S, Bussey HJ. Patterns of mucus secretion in the colonic epithelium in familial polyposis. Investigative Cell Pathology 1980;3:329-43.

14 Habib NA, Dawson PM, Krausz T, et al. A study of histochemical changes in mucus from patients with ulcerative colitis, Crohn's disease, and diverticular disease of the colon. Dis Colon Rectum 1986;29:15-17.

15 Ehsanullah M, Filipe MI, Gazzard B. Mucin secretion in Ehsanullah M, Filipe MI, Gazzard B. Mucin secretion in
inflammatory bowel disease: correlation with disease activity and dysplasia. Gut 1982;23:485-9.

16 Dawson PA, Filipe MI. An ultrastructural application of silver methenamine to the study of mucin changes in the colonic mucosa adjacent to and remote from carcinoma. Histochem $\mathcal{F}$ 1976;8:143-58.

17 Balague C, Audie JP, Porchet N, Real FX. In situ hybridisation shows distinct patterns of mucin gene expression in normal, benign, and malignant pancreas tissue. Gastroenterology 1995;109:953-64

18 Ho SB, Niehans GA, Lyftogt C, et al. Heterogeneity of mucin gene expression in normal and neoplastic tissues. Cancer Res 1993;53:641-51.

19 Chang SK, Dohrman AK, Basbaum CB, et al. Localisation of mucin (MUC2 and MUC3) messenger RNA and peptide expression in human normal intestine and colon peptide expression in human normal in

20 Chang SK, Park ES, Chung WS, et al. Expression of MUC2, MUC3 apomucins and mRNA in ulcerative colitis [abstract]. Gastroenterology 1994;106:A662. 